\title{
Behandlungsrealität bei der Periimplantitis-Prophylaxe
}

Das Aktionsbündnis gesundes Implantat veranstaltete kürzlich eine Umfrage und erhob interessante Daten.

Das Aktionsbündnis gesundes Implantat hat 127 Zahnarztpraxen zur Prophylaxe von periimplantären Erkrankungen befragt. Die Ergebnisse sind größtenteils erfreulich, zeigen aber auch einige Defizite - besonders bei den Themen der präoperativen Patientenaufklärung. Das Aktionsbündnis konnte anhand der Umfrage weitere zentrale Ziele für eigene Projekte zur Förderung der periimplantären Gesundheit identifizieren.

\section{Implantate boomen}

Immerhin 30\% der weiblichen und $57 \%$ der männlichen Zahnmediziner haben sich den Arbeitsschwerpunkt Implantologie gesetzt ${ }^{1}$. Gleichzeitig ist die Implantologie mit einem verhältnismäßig hohen Risiko für Patienten verbunden: Eine europäische Konsensusstudie aus dem Jahr 2006 diagnostiziert an über $50 \%$ der Implantate eine periimplantäre Mukositis und an 12 bis $40 \%$ der Implantate eine Periimplantitis².

\section{Patientenaufklärung}

In den befragten Praxen wird sich für die Patientenaufklärung größtenteils erfreulich viel Zeit genommen. Über zwei Drittel gaben an mindestens 10 bis 20 min Zeit für die Aufklärung vor einer Implantation aufzuwenden. Nur

IDZ-Information Nr. 1/10. Micheelis, W., Bergmann-Krauss, B., Reich, E. Rollenverständnisse von Zahnärztinnen und Zahnärzten in Deutschland zur eigenen Berufsausübung - Ergebnisse einer bundesweiten Befragungsstudie, S. 23.

2 Lindhe J, Meyle J: Peri-implant diseases: Consensus report of the Sixth Euro-

Quelle: pean Workshop on Periodontology, Group
$5 \%$ der befragten Praxen klären in maximal 10 min auf. Da bei der Patientenaufklärung über die Hälfte der befragten Praxen das Assistenzpersonal hinzuzieht, will das Aktionsbündnis zukünftig noch gezielter diese Personengruppe ansprechen, um so Patienten schon präoperativ bestmöglich auf die Implantatpflege vorzubereiten.

Am häufigsten werden die Patienten bei der Aufklärung über mögliche Therapiealternativen, den Behandlungsablauf und die Initialkosten einer Implantation informiert. Die Notwendigkeit der sorgfältigen Implantatpflege nach Einheilung, eine gute Mundhygiene präoperativ, Risikofaktoren für periimplantäre Infektionen und die Gefahr und Häufigkeit periimplantärer Infektionen standen hingegen am wenigsten im Fokus der befragten Praxen.

\section{Implantatplanung}

Bei der Implantatplanung mit bildgebender Diagnostik gibt es große Unterschiede zwischen den befragten Praxen. Ein Großteil nutzt bereits digitales Röntgen, Filmaufnahmen sind aber noch immer sehr verbreitet. An Platz eins der Hilfsmittel für die Implantatplanung steht die digitale Panoramaaufnahme, an zweiter Stelle die Planung auf dem Modell und an dritter Stelle die 3D-Aufnahme.

\section{Häusliche Prophylaxe}

Erfreulicherweise kommen in den befragten Praxen praktisch alle Patienten mindestens zwei mal jährlich zur Implantatprophylaxe, in 20\% der Fälle sogar quartalsweise. Die Mitarbeit der Patienten bei der Implantatprophylaxe wird von knapp $60 \%$ der teilnehmen- den Praxen als gut oder sehr gut bewertet. Allerdings schätzen auch $29 \%$ der Praxen die Compliance lediglich als befriedigend ein, $9 \%$ sogar als ausreichend oder mangelhaft. Hier zeigt sich für das Aktionsbündnis die Notwendigkeit der Patientenmotivation für die Implantatprophylaxe.

\section{Professionelle Prophylaxe}

Bei der Durchführung der Implantatprophylaxe wird am häufigsten mit Handinstrumenten aus Kunststoff oder Titan gearbeitet. Über die Hälfte aller Praxen nutzt für die Implantatprophylaxe Luft-Pulver-Wasserstrahlgeräte. $28 \%$ der Praxen verwenden für die Implantatprophylaxe Chlorhexidin-Präparate. $28 \%$ der befragten Praxen reinigen Implantate mit Schallscalern, 35\% mit Ultraschallscalern (Mehrfachnennung war möglich). Am häufigsten gaben die befragten Praxen an, mit der Methode Piezon zu arbeiten.

\section{Zukünftige Projekte}

Ziele dieser nicht-repräsentativen Umfrage zur Behandlungsrealität bei der Prophylaxe periimplantärer Erkrankungen des Aktionsbündnisses gesundes Implantat waren die Evaluation von Defiziten in der Periimplantitis-Prävention, die Erhebung eines Status quo als Grundlage für kontinuierliche Erhebungen in den kommenden Jahren und die Identifizierung konkreter Ansatzmöglichkeiten für weitere Projekte des Aktionsbündnisses gesundes Implantat.

Stomatologie 2015 - 112:122

DOI 10.1007/s00715-015-0027-8

Online publiziert: 21. April 2015

C Springer-Verlag Wien 2015 